\title{
PAPR Reduction Based on Proposed Rotating Phase Shift Technique in MC-CDMA Using FPGA
}

\author{
Ali Kareem Nahar ${ }^{1,2}$, Kamarul H. Bin Gazali1 \\ ${ }^{1}$ Faculty of Electrical and Electronic Engineering, University Malaysia Pahang, Pekan, Malaysia \\ ${ }^{2}$ Department of Electrical Engineering, University of Technology, Baghdad, Iraq \\ Email: alikareemeng@yahoo.com
}

Received 17 June 2015; accepted 14 July 2015; published 17 July 2015

Copyright (C) 2015 by authors and Scientific Research Publishing Inc.

This work is licensed under the Creative Commons Attribution International License (CC BY).

http://creativecommons.org/licenses/by/4.0/

(c) (i) Open Access

\begin{abstract}
FPGA implementation used of Rotating phase shift (RPS) for peak-to-average power ratio (PAPR) reduction in Multi Carrier Code Division Multiple Access (MC-CDMA) signals. Because, MC-CDMA is still suffering from PAPR which is a major drawback in most of the multi carrier communication systems. In addition, the implementation of the system in an FPGA becomes more flexible and scalable. It eliminates the search for optimum phase factors from a given set, which manifests improved PAPR at reduced computational complexity as compared to conventional PTS and SLM. The amplitude of the signal is reduced by rotating each of the partially transmitted sequence anticlockwise by a $\pi / 2$ degree and the peak power is reduced by circularly shifting the quadrature component of the partially transmitted sequence after phase rotation. A brief description of PTS, SLM is compared with the RPS, which best reduces PAPR from PTS and SLM. It is also presented that VHDL code of the RPS is designed by Xilinx ISE 14.1 implements of FPGA. The peak-to-average power ratio performance of the proposed method has been investigated.
\end{abstract}

Keywords

MC-CDMA, PAPR, RPS and FPGA

\section{Introduction}

The 4G wireless technology has approved MC-CDMA transmission supporting high data rate communications [1]. In the MC-CDMA, orthogonal codes are used to spread symbols of users and combine them in the frequency domain; this results in a comparatively low symbol rate and non-selective fading in each subcarrier [2]. But, the 
MC-CDMA systems are facing problems cast by PAPR due to the nature of the multicarrier OFDM and CDMA [3]. The high PAPR drives the power amplifier to operate in the nonlinear area that causes distortions between inter-modulation and out-of-range radiation. Therefore, it is highly essential to reduce PAPR. In addition, several techniques have been proposed like clipping and filtering, coding, active constellation extension, Tone Injection (TI), Tone Reservation (TR), partial transmit sequence (PTS), and selected mapping (SLM) [4]. Among all these approaches, SLM is considered to be appropriate to reduce the PAPR, whereas its computational complexity is very high [5] [6].

In [7], the authors have proposed a lower complexity PAPR reduction scheme, which at the transmitter side a single IFFT block is used without any sub-block partitioning or phase factors. FPGA implementation of DS-CDMA transmitter has been proposed in [8], which research complicated two phases-simulation and synthesis of the Verilog codes. The Xilinx Synthesis Technology (XST) of Xilinx ISE 12.3 tool was used for synthesis of the transmitter and simulates a design's reaction to different stimuli. In [9], the authors suggested a low complexity PTS, which includes cyclic shifting of time domain sequences and combining them which leads to reduced computational complexity. In addition, Low Complexity VLSI Architecture for DS-CDMA Communication System uses FPGA [10]. In [11], the error control code makes each sequence more random and reduces the probability of in phase addition of sub-carriers by interleaved. Thus, it is an improved SLM method with n-tuple PAPR control bits followed by an interleaved and an error control code. The PAPR reduction in the MC-CDMA system increases the probability of PAPR reduction.

In this present paper, the use of phase factors is eliminated, which is originally employed in SLM and PTS, by introducing the method of rotating each time domain sub-block symbol by a predetermined degree and circular shifting of partially transmitted quadrature phase components. Moreover, the Very High Speed Integrated Circuits Hardware Description Language (VHDL) implementation of Rotating Phase shifted technique is achieved where parallel processing of symbols is carried out instead of serial processing. Furthermore, the multiplicative complexity is reduced by shift-and-add algorithm. VHDL implementation of PAPR calculation is also performed which provides the PAPR of the respective symbol transmitted.

This paper is organized as: Section 2 discussed a brief review of PAPR in MC-CDMA system. It proposed method Rotating Phase Shift display in Section 3. In Section 4, RPS-PAPR is implemented in Xilinx ISE 14.1 using VHDL. Finally, results of simulation performed are provided in Section 5. Section 6 presents the concluding remarks.

\section{The PAPR of an MC-CDMA Signal}

The MC-CDMA is a multi-carrier transmission, which has a high data rate and greater flexibility for voice, data, and video and internet services for future wireless systems. This is multiplied by the first of the original data load, with the spreading code and then the chips of spread data are modulated onto orthogonal subcarriers [12]. Figure 1 shows the MC-CDMA signal generation in transmitting side of a complex data symbol an assigned to user h. In the transmitter of data symbol $a^{h}$ is first multiplied with the user specific spread code by $=$ $\left[b_{1}^{h}, b_{2}^{h}, \cdots, b_{M-1}^{h}\right]^{\mathrm{T}}$ of spread factor $M$. The spread code $c^{h}$ obtained after spreading can be given in vector scheme as:

$$
c^{h}=a^{h} b^{h}=\left[C_{1}^{h}, C_{2}^{h}, \cdots, C_{M-1}^{h}\right]^{T}
$$

The $\mathrm{c}^{h}$ is converted to parallel $C_{m}^{h}$, where $m=0,1, \cdots, M-1$, and modulated onto $\mathrm{M}$ subcarriers followed by IDFT of size $N=1 \times M$ to obtain a multi-carrier spread spectrum signal. A time domain baseband transmission signal $x^{h}(t)$, after IDFT, for one MC-CDMA symbol, $0 \leq t \leq T_{s}$, has:

$$
x^{h}(t)=\sum_{m=1}^{M} \sum_{h=1}^{H} a^{h} b_{m}^{h} \mathrm{e}^{j 2 \pi(m-1) t / T_{s}}
$$

where $T_{s}$ is the MC-CDMA symbol period and $\mathrm{H}$ the total number of users.

Formerly, the PAPR [13] of MC-CDMA signals $x(t)$ is defined as the ratio between the maximum instantaneous power and average output power

$$
\mathrm{PAPR}=\frac{P_{\text {peack }}}{P_{\text {average }}}=\frac{\max _{0 \leq n \leq m-1}\left(|x|^{2}\right)}{E\left\{\left|x^{2}\right|\right\}}
$$




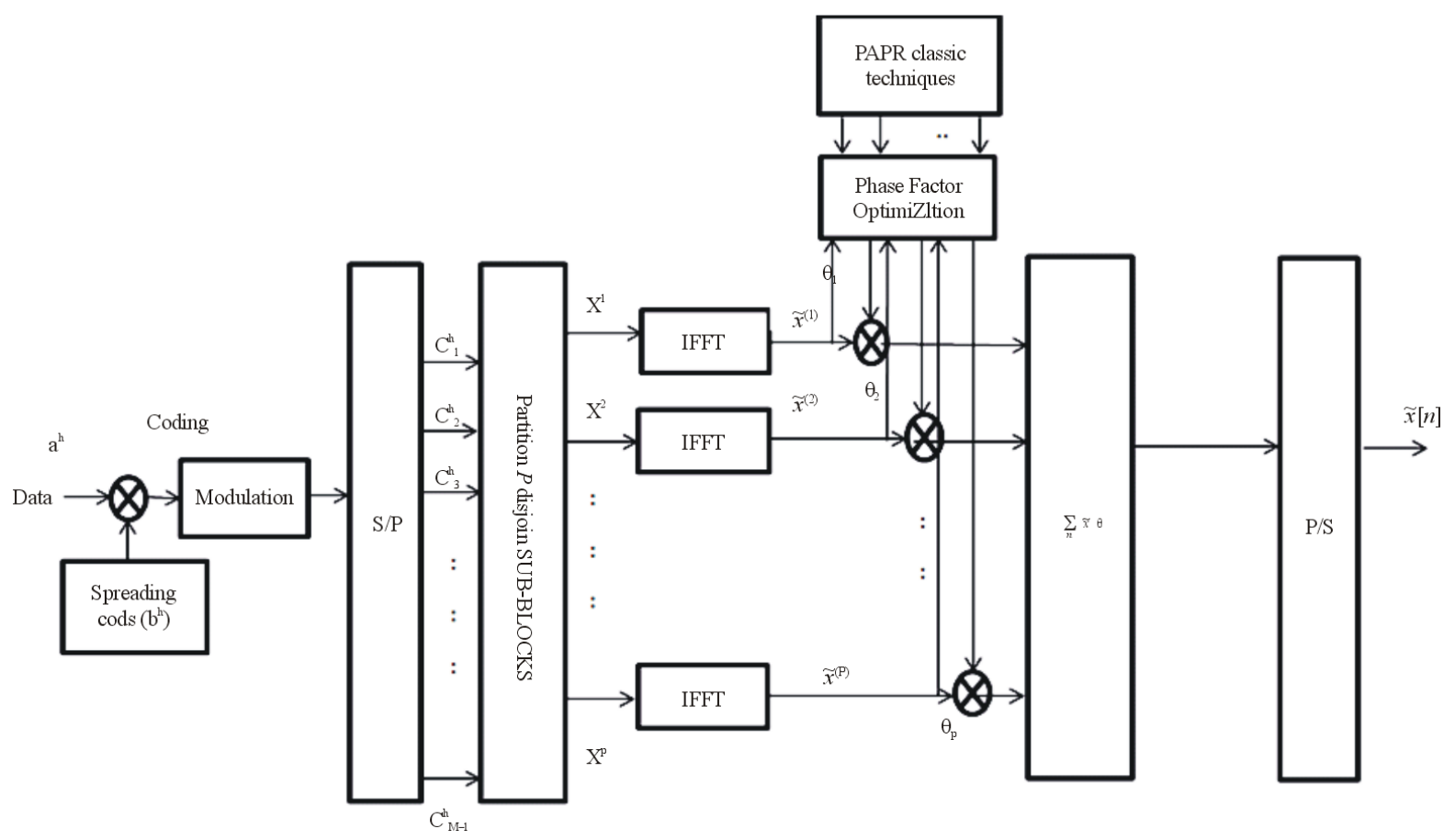

Figure 1. Block diagram of MC-CDMA, transmitted side.

$P_{\text {average }}$ can be calculated as:

$$
P_{\text {average }}=\frac{1}{T_{s}} \int_{0}^{T_{s}}|x(t)|^{2} \mathrm{~d} t=\frac{1}{N} \sum_{n=0}^{N-1} E\left\{\left|\sum_{h=1}^{H} x^{h}(n)\right|^{2}\right\}
$$

where $N$ is the number of sub-carriers and $E\{$.$\} denotes expectation. Also, PAPR in the discrete time domain$ can be expressed as:

$$
\operatorname{PAPR}_{\max }=10 \log _{10}(N)(\mathrm{dB})
$$

As one of the characteristics of the PAPR, which bears stochastic characteristics in MC-CDMA systems, often can be expressed in terms of Complementary Cumulative Distribution Function (CCDF). Which is described as the probability of the PAPR exceeding a certain level w [14] [15], that can calculate as:

$$
\operatorname{CCDF}(\mathrm{PAPR})=\operatorname{prob}\{\mathrm{PAPR}>w\}=1-\left(1-\mathrm{e}^{-w}\right)^{N}
$$

where, $N$ total number of sub-carrier and $w$ is the level of exceed.

\section{Proposed Method}

RPS takes away the use of phase factors and reduces PAPR. After multiple data by spreading code, then modulation, the data symbols are partitioned into sub-blocks which generate the frequency domain symbols [16]. These frequency domain symbols are converted to time domain symbols by N-point IFFT operation on each sub-block. Figure 2 shows the block diagram of the transmitter system of proposed RPS-PAPR. Now, instead of combining phase factor and the rotation phase of the work in phase and beating by rotating phase is employed, to suppress the amplitude of a signal. Phase rotation adjusts the amplitude of the samples, but the power of the samples has not changed. Moreover, the quadrature components of the output samples of symbols after rotation stage and turned carousel by the equation of shift where the transformation of each quadrature component $p$ times. In a joint operation of the phase rotation and circular shift results in reducing PAPR.

On the other hand, the support sets of $X_{p}$ 's are disjoint. The sub-vector $x_{p}=\left[x_{p, 0} x_{p, 1} \cdots x_{p, N-1}\right]^{\mathrm{T}}$ is created using apply IFFT to each symbol sub-vector $X_{p}$, also known as a sub-block. Each sub-vector $x_{p}$ is 


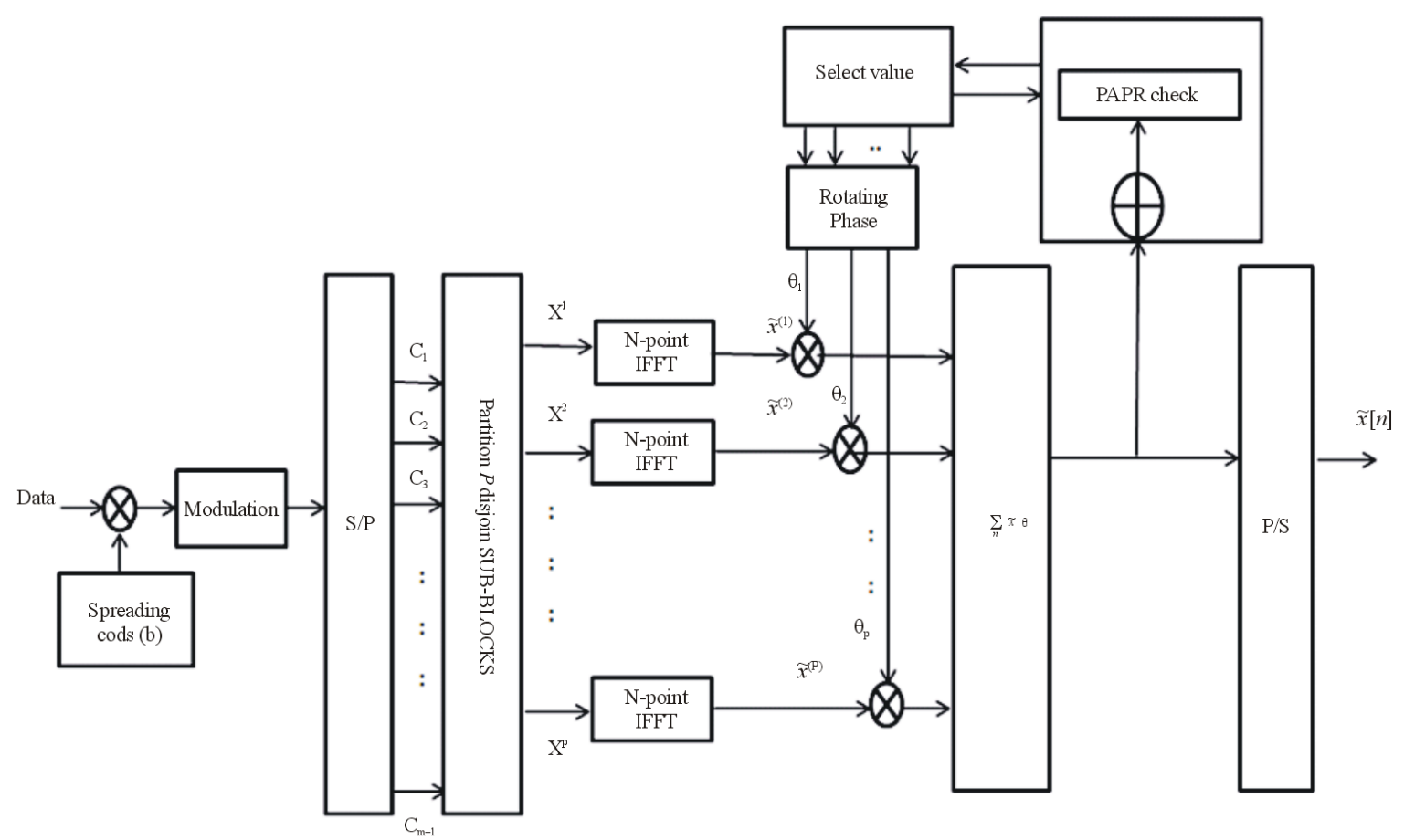

Figure 2. Bloc diagram of the MC-CDMA with RPS PAPR reduction technique.

then multiplied by a valid phase shift $\theta=\left[\theta_{1}, \theta_{2}, \cdots, \theta_{P+1}\right]$ chosen from a given symbols $\xi$, that is generally $\xi=\{ \pm 1\}$ or $\xi=\{ \pm 1, \pm j\}$, where $\xi$ the rotating facto. Also can be shifted the phase on the circumference of a circle to any value such as $\pi / 4$ that is shown in Figure 3. Similarly, this results in a rotating MC-CDMA signal vector $x^{w}=\left[\begin{array}{llll}x_{0}^{w} & x_{1}^{w} \cdots & x_{N-1}^{w}\end{array}\right]^{\mathrm{T}}$ given as

$$
x^{w}=\sum_{p=1}^{P} r_{p}^{w} x_{p}
$$

where,

$$
r^{w}=\left[\begin{array}{llll}
r_{1}^{w} & r_{2}^{w} & \cdots & r_{p}^{w}
\end{array}\right], 1 \leq w \leq W, \text { and } W=|\xi|^{p+1}
$$

The PAPR of $x^{w}$ is computed for rotating vectors and compared, which has the minimum PAPR is selected for transmission. The index $\tilde{w}$ of the corresponding rotating vector $r^{\tilde{w}}$ is selected according to

$$
\tilde{w}=\arg \min _{1 \leq w \leq W} \max _{0 \leq n \leq N-1}\left|\sum_{p=1}^{P} r_{p}^{w} x_{p, n}\right|
$$

The receiver must know the index information to recover the original input vector. The PAPR reduction performance and the computational complexity of the RPS structure depend on the method of sub-block separating. Furthermore, a search is a strategy that works well on optimization problems with the minimize PAPR show in Algorithm. 1 the search algorithm For algorithm, let $X$ be the MC-CDMA signal and $\theta$ be a set of valid phase shifts [17].

Algorithm 1: GVS Search

* Let: $\theta=\left\{\theta_{1}, \theta_{2}, \cdots, \theta_{P}\right\}$ be phase at partition limit.

* Require: $X>0, P>0, k>0, \varphi>0$;

* Inialize: $x_{o p}(t)=X[0] ; \theta=\varphi_{M}, R_{m}=0$.

* For $p=1: P$ do

* $X_{p}[n]=\left\{\begin{array}{l}X[n], n=r_{p-1}+1, \cdots, r_{p} \\ 0, \quad \text { otherwise }\end{array}\right.$ 
- $\quad$ For $m=1: M$ do

$$
\begin{aligned}
& R_{m+1}=R_{m}+\frac{\left(r_{p}+1\right)\left(r_{p-1}\right)}{2} \\
& X_{p}^{m}[n]=X_{p}[n] \mathrm{e}^{\left(j\left(\theta_{p}+\frac{R_{m}}{R_{m+1}-R_{m}}\right) \varphi_{m}\right)} ;
\end{aligned}
$$

- IF $n<r_{p}$ then

$$
x_{0}^{m}(t)=i f f t\left(X_{p}^{m}\right)
$$

- Else empty $R_{m+1}$, otherwise $n$

- End

$$
\begin{aligned}
& m_{o p}=\arg \min _{m=1: M}\left(\operatorname{Max}_{0 \leq t \leq \tau}\left(\left|x_{o p}(t)+x^{m}(t)\right|\right)\right) ; \\
& x_{o p}(t)=x_{o p}(t)+x^{m_{o p}}(t) ; \\
& \theta_{P}=\theta_{P-1}+\varphi_{m_{o p}} ;
\end{aligned}
$$

* End

* Return $x_{o p}(t), \theta, R_{m}$

In addition, to define the effect of each factor is compared to the performance of the RPS algorithm by selecting one of the factors are constant and change the rest of the factors in order to measure the effectiveness of that factor in the reduction of PAPR and then change with all cases the same method and each parameter triple $\left(N_{R}, Z_{\theta}, R_{p h}\right)$. As a zero phase shift is always one of the valid phase shifts, the relationship of these factors can be defined as:

$$
\left(1-\frac{\xi^{2 N_{R}}}{N_{R}}\right) Z_{\theta}=R_{p h}
$$

where $N_{R}$ is the number of valid phase shifts, $\xi$ the rotating factor $R_{p h}$ the rotate a range of possible phase shifts, $M$ number of partition phase and $Z_{\varnothing}$ the step size of the phase shifts. Phase delay values for RPS algorithm are in $\left(R_{p h}, 0\right)$. This can be illustrated calculating the previous equation, taking into account all or most of the possible cases, according to the following shown in Table 1. Besides, Table 2 turns of the other cases with phase shift e.g. $-\pi / 4$.

\section{VHDL Implement Based on the RPS-PAPR Reduction}

Implement the RPS-MC transmitter system and its peak to average power ratio calculation in VHDL. The archi-

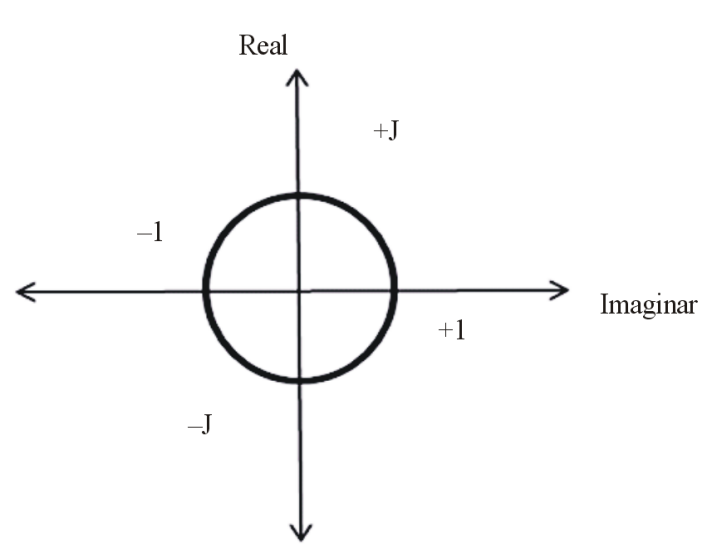

(a)

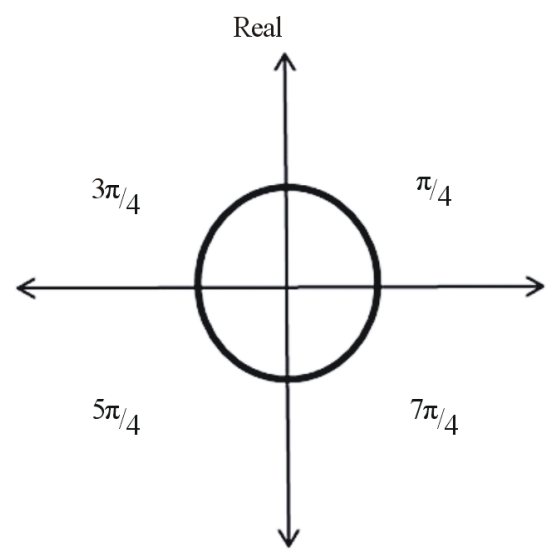

(b)

Figure 3. (a) Rotate magnitude constant $\xi$; (b) Phase shift by $\pi / 4$. 


\begin{tabular}{ccccc} 
Table 1. Phase lags $\left(R_{p h}\right)$ at $\xi= \pm j$. \\
\hline \multicolumn{5}{c}{$Z_{\theta}$} \\
$N_{R}$ & $\pi / 2$ & $\pi$ & $3 \pi / 2$ & $2 \pi$ \\
\cline { 2 - 5 } & $\pi$ & $2 \pi$ & $\pi$ & $2 \pi$ \\
2 & $\pi / 4$ & $\pi / 2$ & $3 \pi / 4$ & $\pi$ \\
3 & $2 \pi / 3$ & $4 \pi / 3$ & $2 \pi$ & $\pi / 3$ \\
4 & $3 \pi / 8$ & $3 \pi / 4$ & $9 \pi / 8$ & $3 \pi / 2$ \\
\hline
\end{tabular}

\begin{tabular}{ccccc} 
Table 2. phase lags & $\left(R_{p h}\right)$ & with phase shift & $-\pi / 4$ \\
\hline \multirow{5}{*}{$N_{R}$} & \multicolumn{4}{c}{$Z_{\varnothing}$} \\
\cline { 2 - 5 } & $\pi / 4$ & $3 \pi / 4$ & $5 \pi / 4$ & $7 \pi / 4$ \\
\hline 1 & $\pi / 2$ & $3 \pi / 2$ & $\pi / 4$ & $3 \pi / 2$ \\
2 & $\pi / 8$ & $3 \pi / 8$ & $5 \pi / 8$ & $7 \pi / 8$ \\
3 & $\pi / 3$ & $\pi$ & $5 \pi / 3$ & $\pi / 3$ \\
4 & $3 \pi / 16$ & $9 \pi / 16$ & $15 \pi / 16$ & $21 \pi / 16$ \\
\hline
\end{tabular}

tecture proposed in this paper was coded in VHDL and then simulated and synthesized in Xilinx ISE 14.1. The computational complexity which arises due to IFFT operation is reduced by its VHDL implementation [18]. Where, the MC-CDMA signal partition according rotation factor $R_{p s}$ the rotate a range of possible phase shifts shown in Table 3, for parallel processing of the time domain symbols, a multiplexer is incorporated where the select inputs are selected by a select maximum value. In addition, the PAPR calculation of this MC-CDMA symbol is calculated and the symbol with minimum peak-to average power ratio is transmitted. The same can be evaluated in VHDL as per the block diagram shown in Figure 4. At this point, the operations are performed individually on Rotating Phases Shift components. The inputs are taken in integer representation upon which squaring, adding and division operations are performed. Figure 5 shows the overall for RPS-PAPR calculation of an MC symbol after implementing in FPGA that shows the input IFFT for 64-bits, real and imaginary, then the output after PAPR reduction with enable control in order to build RTL circuits. Moreover, Figure 5 shows the PAPR architecture in the ISE 14.1 RTL circuit for more details.

\section{Simulation Results}

Simulations were performed to compare the performance of PAPR reduction in MC-CDMA system SLM, PTS and RPS at phases $3 \pi / 2$ and $3 \pi / 8$, with $N=64$ subcarriers. The CCDF plots of the PAPR of the MC-CDMA signals in Figure 6 are shown for four users. Number of partitions for proposed technique RPS and classic PTS is four while predetermined codes for SLM method take 32 and 64. The performances for proposed at both shift registers is best an author's technique as shown in Figure 7. The PAPR reduction performance is measured using simulation parameters for MC-CDMA as in Table 4

RPS-PAPR calculation of the MC-CDMA system is calculated and the symbol with minimum peak-to average power ratio is transmitted. Table 5 represents the resources utilization summary of the RPS. In addition, Figure 8 shows the test-bench waveform for PAPR calculation of an MC-CDMA symbol. It can be observed that for each transmitted symbol a PAPR is calculated. Moreover, the test-bench waveform of the transmit side as shown in Figure 9.

\section{Conclusion}

RPS technique is achieved, which verifies the best PAPR reduction as compared to traditional SLM and PTS techniques. Additionally, the application is parallel processing and pipeline of symbols by implementation in 
Table 3. Control registers values.

\begin{tabular}{ccc}
\hline $\boldsymbol{R}_{\boldsymbol{p h}}$ & $\boldsymbol{R}_{p s}$ With shift $\pi / 4$ & Register Value \\
\hline$\pi / 2$ & $\pi / 4$ & 01 \\
$\pi$ & $3 \pi / 4$ & 10 \\
$3 \pi / 2$ & $5 \pi / 4$ & 11 \\
$2 \pi$ & $7 \pi / 4$ & 00 \\
\hline
\end{tabular}

Table 4. MC-CDMA simulation parameters.

\begin{tabular}{cc}
\hline Parameters & Value \\
\hline Number of transmitting bits & 2000 \\
No. Subcarriers $N$ & 64 \\
No. of Users $H$ & 4 \\
Modulation & $32-\mathrm{QAM}$ \\
IFFT\&FFT size & 128 \\
Additive noises & $20 \mathrm{~dB}$ \\
Spading code type & Gold code \\
No. Partitions $P$ & 4 \\
Predetermined codes $C_{m}$ & 16,32 \\
\hline
\end{tabular}

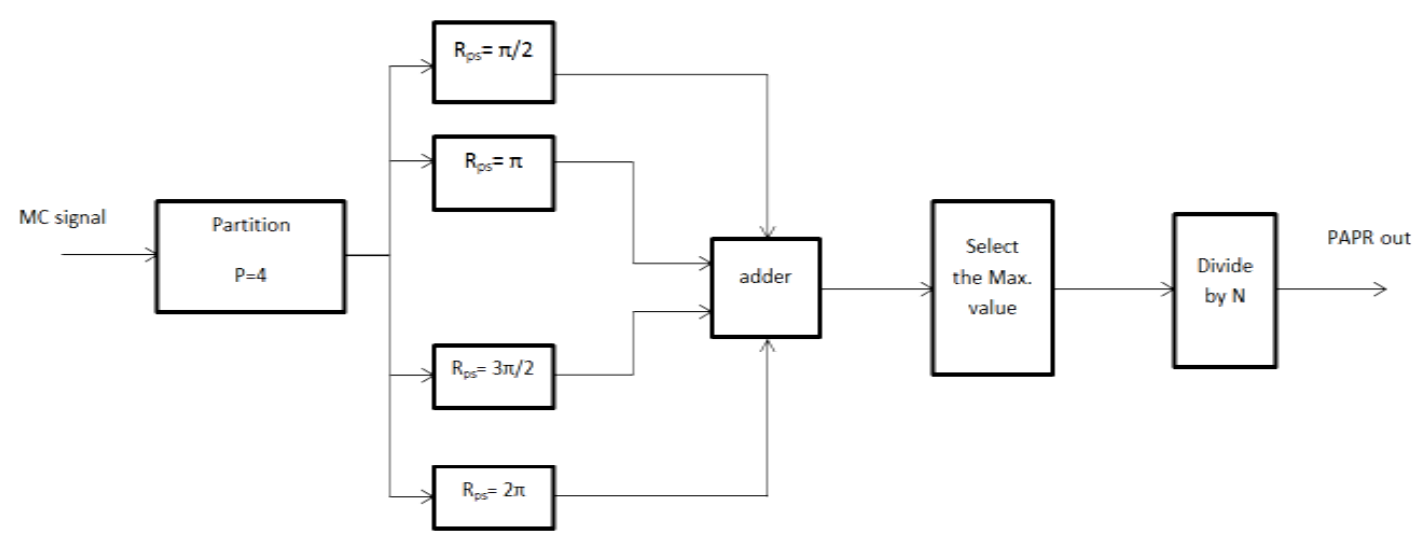

Figure 4. Block Diagram for PAPR calculation in VHDL.

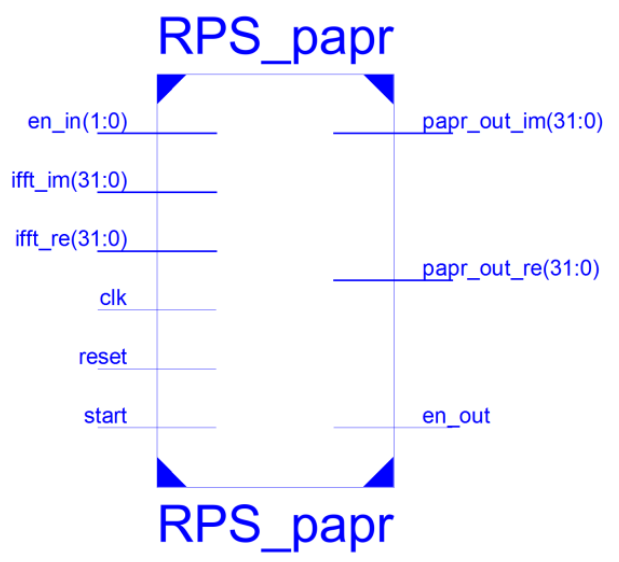

(a) 


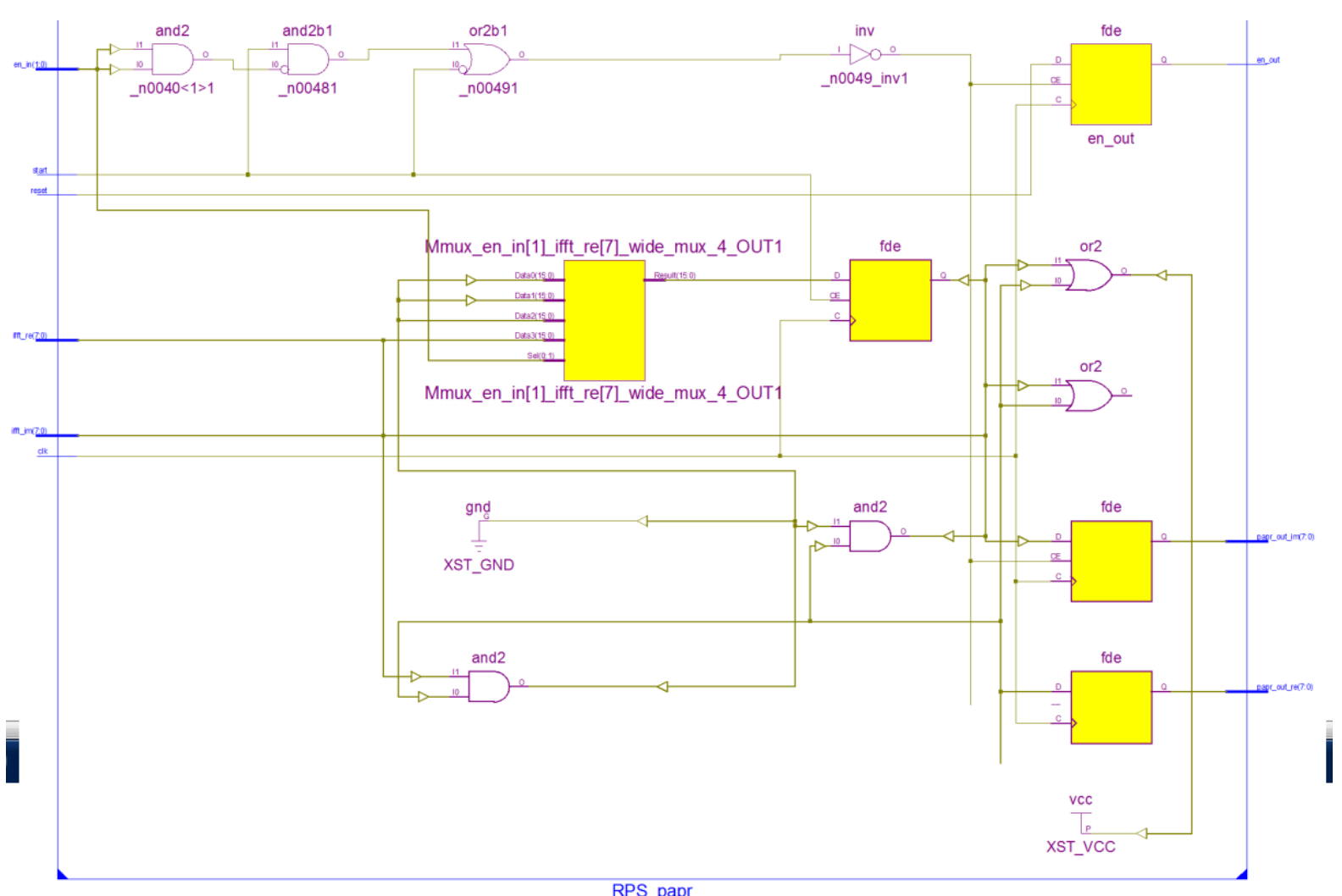

(b)

Figure 5. Top level view (a) with all inputs \& outputsof the RPS-PAPR and (b) details for RTL circuits.

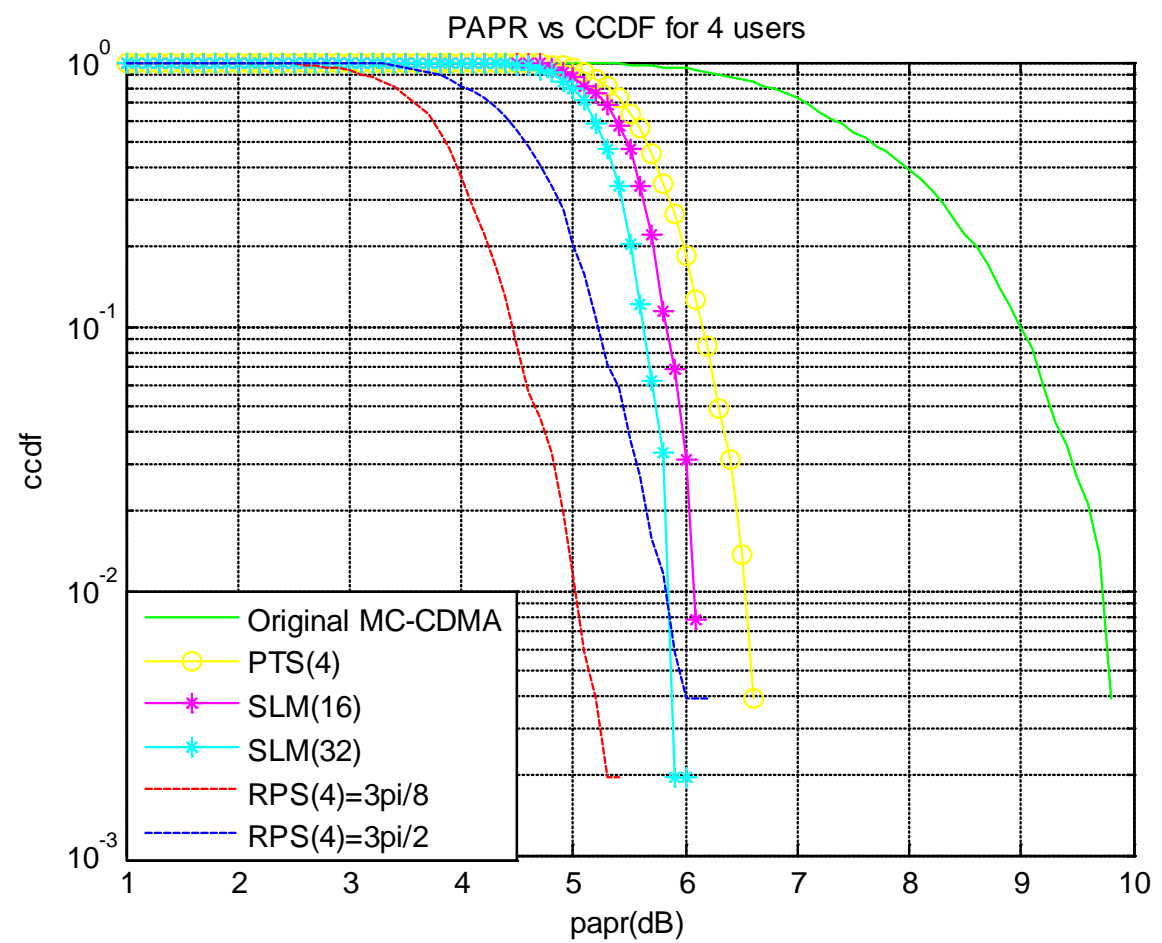

Figure 6. A CCDF of the PAPR of 4 users MC-CDMA signals compares an SLM $C_{m}=16, C_{m}=32$ and PTS and RPS, P $=4$, 32-QAM. 


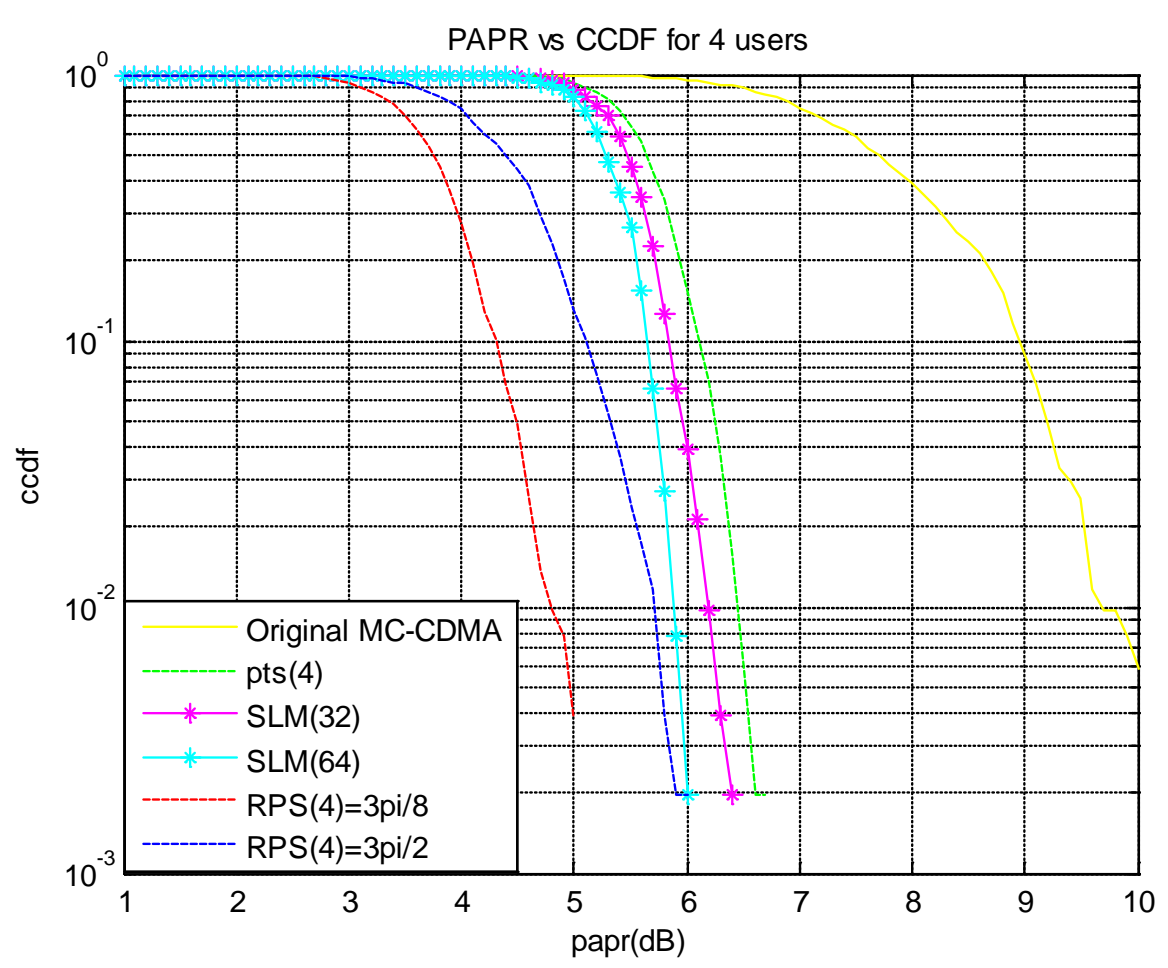

Figure 7. A CCDF of the PAPR for 4 users of MC-DMA signals compares an SLM $C_{m}=16, C_{m}=32$ and PTS P $=4$, and RPS $R_{p h}=3 \pi / 2, R_{p h}=16 \pi / 21$.

Table 5. Resources utilization summary.

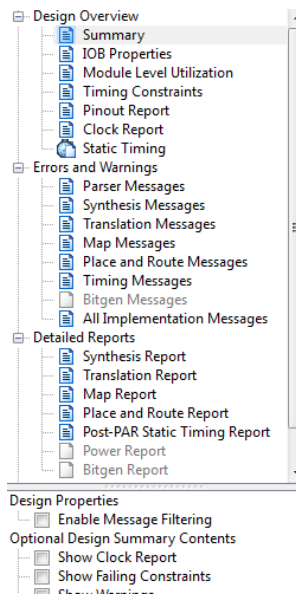

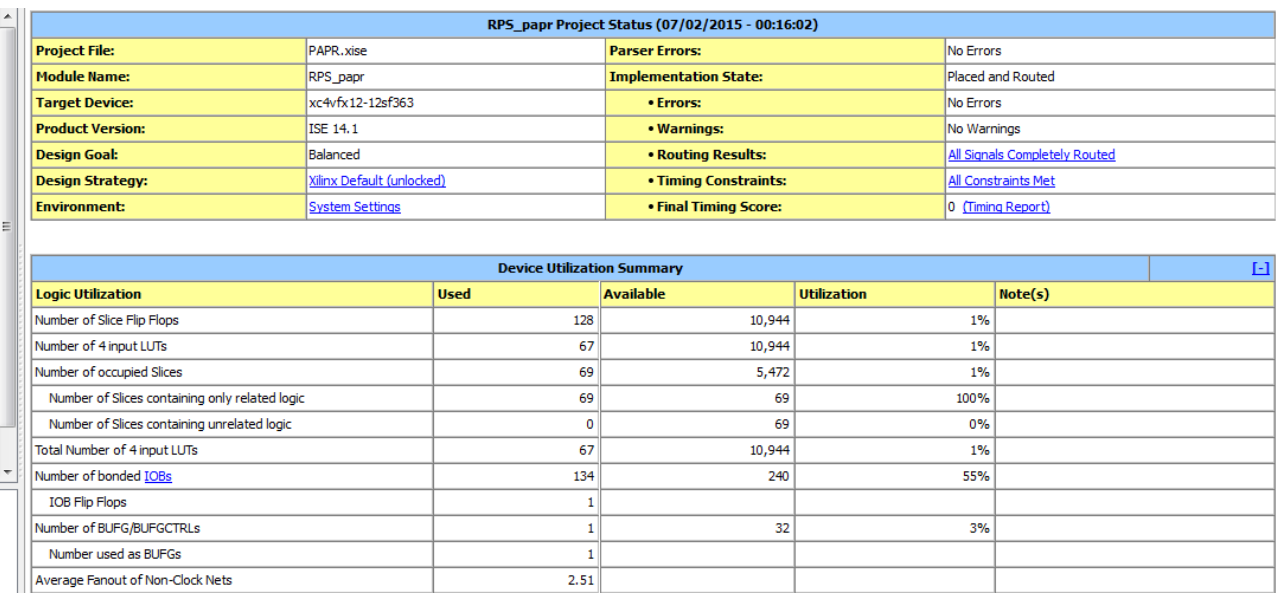
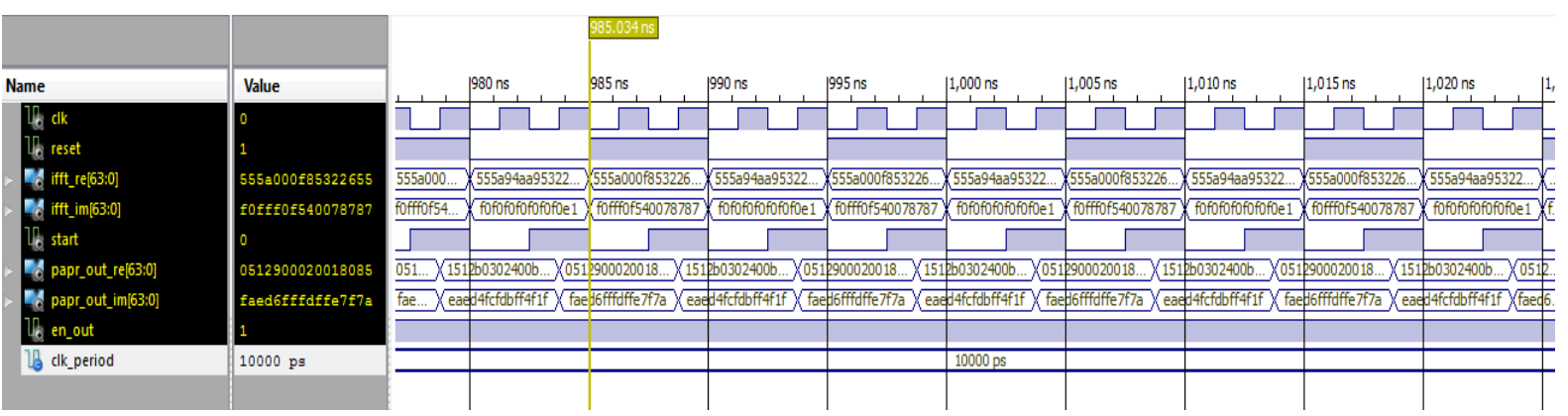

Figure 8. PAPR calculation test-bench waveform. 


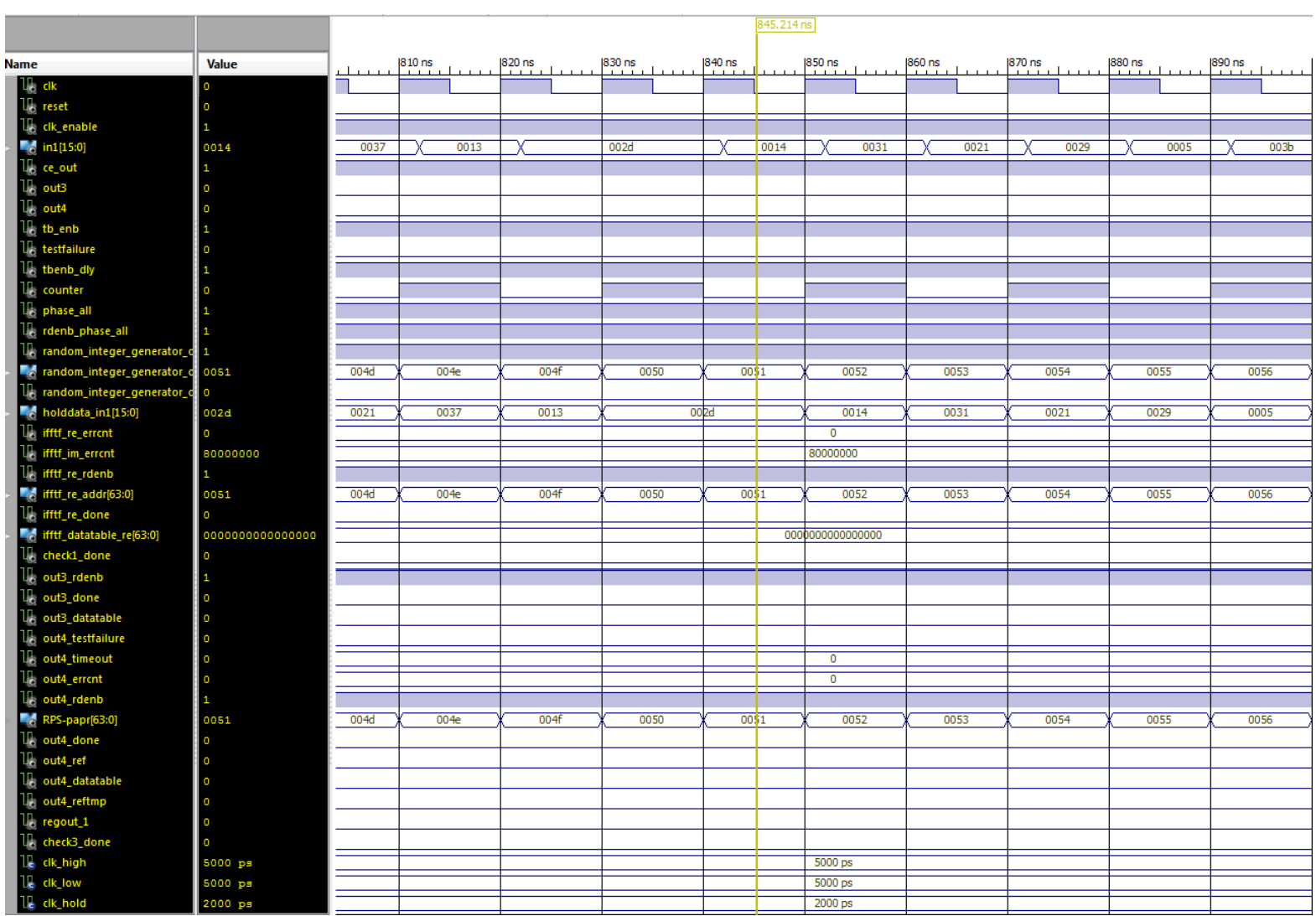

Figure 9. Transmitting side in MC-CDMA System test-bench waveform.

VHDL, which reduces the complexity by eliminating the difficult multiplication. Matlab simulations are done for 1000 samples of the MC-CDMA system with $\mathrm{N}=64$ subcarriers to plot the CCDF, which shows that RPS is the best method compared with the ways in various cases. The same idea is implemented in VHDL to calculate PAPR, and it can also be a simulation of subcarriers equal to 16 and 32 using the same process. This system eliminates the phase combined factor with lower PAPR better.

\section{References}

[1] Mahore, A.S., Singh, B. and Tanwar, P.S. (2014) A Review 4G System QAM MC-CDMA Higher Data Rates Transmission Systems in Impulsive Noise. International Journal of Electrical, Electronics Computer Engineering, 3, 177 182.

[2] Sarala, B., Venketeswarlu, D.S., Bhandari, B.N. and Srinivas, B. (2014) Discrete Transforms based MC-CDMA PAPR Reduction using MECCT and Bit Error Rate Performance Analysis over Mobile Radio Channels. Proceedings of International Conference on Recent Trends in Information, Telecommunication and Computing, ITC, 238-247.

[3] Sarala, B., Venkateswarulu, D.S. and Bhandari, B.N. (2012) Overview of MC-CDMA PAPR Reduction Techniques. International Journal of Distributed and Parallel Systems (IJDPS), 3, 193-206. http://dx.doi.org/10.5121/ijdps.2012.3217

[4] Hernandez, L.A.P. and Otero, M.G. (2009) User Reservation Approach for Peak-to-Average Power Ratio Reduction in MC-CDMA Systems. Proceedings of IEEE 69th Vehicular Technology Conference VTC, Barcelona, 26-29 April 2009, $1-5$.

[5] Xia, J., Li, Y., Zhang, Z., Wang, M., Yu, W. and Wang, S. (2013) A Suboptimal TR Algorithm with Fixed Phase Rotation for PAPR Reduction in MC-CDMA System. IET International Conference on Information and Communications Technologies, Beijing, 27-29 April 2013, 415-420.

[6] Ohkubo, N. and Ohtsuki, T. (2002) A Peak to Average Power Ratio Reduction of Multicarrier CDMA Using Selected Mapping. IEEE 56th Vehicular Technology Conference, 4, 2086-2090.

[7] Eom, S.S., Nam, H. and Ko, Y.C. (2012) Low-Complexity PAPR Reduction Scheme without Side Information for 
OFDM Systems. IEEE Transactions on Signal Processing, 60, 3657-3669. http://dx.doi.org/10.1109/TSP.2012.2191779

[8] Mahbub, T.S., Ahmed, S. and Rokon, I.R. (2011) Transmitter Implementation Using DS-CDMA Technique in FPGA Using Verilog HDL. International Conference on Electrical, Electronics and Civil Engineering (ICEECE), Pattaya, 204-207.

[9] Yang, L., Soo, K., Li, S. and Siu, Y. (2011) PAPR Reduction Using Low Complexity PTS to Construct of OFDM Signals without Side Information. IEEE Transactions on Broadcasting, 57, 284-290.

[10] Amsavalli, A. and Kashwan, K.R. (2012) FPGA Implementation of Low Complexity VLSI Architecture for DS-CDMA Communication System. International Journal of Computer Applications, 42, 27-34.

[11] Ali, S., Chen, Z. and Yin, F. (2015) A Peak-to-Average Power Ratio Reduction Using N-Tuple Selective Mapping Method for MC-CDMA. ETRI Journal, 37, 338-347. http://dx.doi.org/10.4218/etrij.15.0114.0646

[12] Hernández, L.A.P. and Otero, M.G. (2009) User Reservation Approach for Peak-to-Average Power Ratio Reduction in MC CDMA Systems. IEEE Vehicular Technology Conference, Barcelona, 26-29 April 2009, 1-5.

[13] Shinsuke, H. and Ramjee, P. (2003) Principle and History of MCM/OFDM in Multicarrier Techniques for 4G Mobile Communication. Artech House, London.

[14] Yang, D.K., Yu, X., Liu, K.F. and Zhang, Q.S. (2009) Performance Analysis of PAPR in MC-CDMA System. IEEE 1st International Conference on Information Science and Engineering (ICISE), 26-28 December 2009, Nanjing, 26762679.

[15] Sarala, B. and Venkateswarlu, D.S. (2011) MC-CDMA PAPR Reduction Techniques Using Discrete Transforms and Companding. International Journal of Distributed and Parallel Systems, 2, 253-270. http://dx.doi.org/10.5121/ijdps.2011.2622

[16] Saxena, P. and Kumar, S. (2014) Challenges \& Evolution of Next Generation in Mobile Communication Network. International Journal of Advanced Research in Computer Science and Software Engineering, 4, 348-356.

[17] Othman, R.R., Zamli, K.Z. and Nugroho, L.E. (2012) General Variable Strength t-Way Strategy Supporting Flexible Interactions. Maejo International Journal of Science and Technology, 6, 415-429.

[18] Moffo, B.L. and Mbihi, J. (2015) A Novel Digital Duty-Cycle Modulation Scheme for FPGA-Based Digital-to-Analog Conversion. IEEE Transactions on Circuits and Systems II: Express Briefs, 62, 543-547. 